\title{
Using Finite Element Analysis to Assess and Prevent the Failure of Safety Critical Structures
}

\author{
Daniel J. Thomas
}

Submitted: 20 November 2016/Published online: 7 December 2016

(c) ASM International 2016

When designing safety critical structures, it is vital to ensure that integrity and reliability is maintained and that not be compromised throughout the entire life of the application. In yesteryear, designers would progress from a computer-aided design to a fabricated concept prototype, which would then undergo functional testing. At this stage, if the design is determined to be structurally or materially defective, then this required redesign and a subsequent second-stage prototype generated, which results in high costs incurred, time delays and iterations in design cycles.

The automotive industry would once crash-test hundreds of prototype vehicles on the path to developing a final product. This is where finite element analysis (FEA) comes in, allowing a lot more to be learned about materials and structural engineering before the fabrication of the first prototype. During the combined design and FEA process, a complex automotive structure can progress in several design directions, which results in the final product being highly optimised to its environment. For engineering designers FEA modelling is useful to highlight possible design and material issues in any region of the component whilst it is still in its design stage, as shown in Fig. 1.

Further to this, FEA is used to study how a structure will respond to complex stress loading and to predict any possibility of structural failure. Examples of physical effects include repeated impact, strain induced from sudden or prolonged loading, vibration and temperature changes. Each of these factors can be used to build up a variety of condition scenarios that can be assessed. This is important when it comes to assessing fatigue life of the application

D. J. Thomas ( $\square)$

Creation Engineering Group, Llynfi Enterprise Centre, Maesteg, UK

e-mail: daniel.thomas@creationengineering.co.uk which remains the key in-service failure mode for chassis and suspension components.

Under each condition, FEA predicts if the component will fracture, distort, buckle, be susceptible to fatigue or wear out when subjected to stress. It is used to demonstrate that a design will work according to the design requirements for stiffness, safety factor and displacement under load. The immediate benefits of FEA for minimising failure include the following:

- Assessment of and assurance of the product safety factor, which is usually an order to magnitude by two and a half times over the minimum safety factors;

- increased confidence that the design will work as intended when factoring in complex loading patterns; and

- determining quantifiable improvements in part design such as optimising strength, improving weight reduction, enhancing product durability, while at the same time optimising product cost.

The ultimate purpose of FEA simulations is to ensure that component assemblies will function as intended for its entire life. This is particularly the case with components that are joined using welding processes and which are subsequently under high-stress loads as shown in Fig. 2. This applies not only to automotive products, but also to everyday components, from office equipment to household furniture, which all need to be subjected to testing processes in order to comply with safety requirements.

The applications for FEA in the context of design optimisation and lifetime durability prediction include the following:

- Structural integrity testing,

- Fracture testing, 
Fig. 1 Ford Mondeo sub-frame design prior to FEA assessment and the final fabricated component
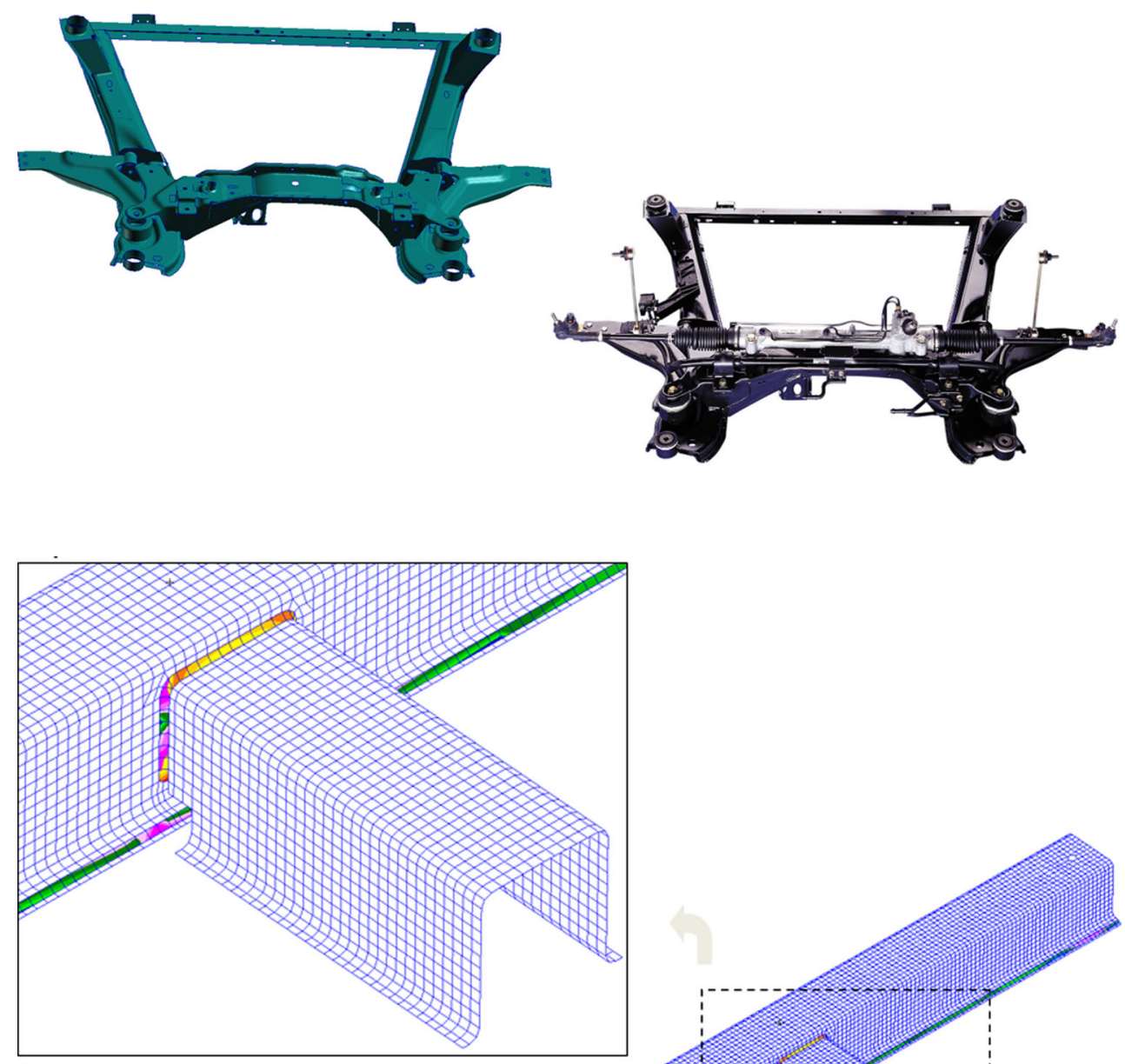

Fig. 2 Weld fatigue damage assessment of two seam welded beams (credit Corus Automotive)
- Fatigue life,

- Pressure testing,

- Loading and stress assessment,

- Vibration or shock impact,

- Thermal loads and temperature change,

- Airflow and fluid dynamics,

- Drop testing.

During the FEA process, a solid body design is separated into many thousands of geometrical elements, collectively called the mesh. Each element is subjected to a mathematical calculation. A processor then assembles each of the elements data set and forms a sequence of results showing how it reacts to thermal or structural loads. Regions of high detail, or high anticipated stress, require higher element density than relatively non-critical areas. It is this increased detail which provides more accurate representations to the real-world performance.
All materials have elastic tolerance limits to the stresses that they can undergo before plastic deformation. For instance, a high-strength steel grade has an 'elastic deformation limit' of 325-385 MPa, where if the component is exposed to stress beyond this amount, then it will not return to its original shape, subsequently there is plastic deformation. If the steel component is further deformed beyond its ultimate tensile strength limit, then the component will break into two parts. Using this example, we can define linear FEA and non-linear FEA:

- Linear analysis models the material in its elastic deformation phase;

- Non-linear analysis is used to model plastic deformation.

In each case, it is critical to ensure that the steel component when it is deformed in service will not be permanently 
deformed. As a result, linear FEA is used to ensure the design results in a product that does not deform. Subsequently, steady-state analysis is carried out to further test for changes in behaviour when a thermal or dynamic structural load is applied as is the case with fatigue.

Contemporarily, there are now numerous commercial software tools that allow for both design and FEA assessments be carried out in a single environment. Subsequently, FEA reduces the reliance on traditional hand calculation, although these are still good to carry out when confirming the FEA results. It is true to say that it is very easy to generate an impressive-looking FEA model, but which also gives an erroneous result. It is therefore useful to perform a few simple hand calculations to get an idea of where the simulation results are as expected or at the very least provide a result to an order of magnitude.

Computer-based analysis of structures is a vital tool to assess and subsequently prevent the failure of safety critical structures. It can be used by all engineers to overcome some of the future complex challenges that we all face everyday. 